\title{
Formulation of Domperidone Microspheres Using a Combination of Locally Extracted Chitosan and Hpmc as Polymers
}

\author{
Stephen Olaribigbe Majekodunmi and Cynthia Chibuzor Uzoaganobi \\ Department of Pharmaceutics and Pharmaceutical Technology, Faculty of Pharmacy, University of Uyo, P.M.B 1017, Uyo, \\ Akwa-Ibom State, Nigeria
}

\begin{abstract}
Commercially available domperidone -a D2 receptor antagonist- is an immediate release formulation which has never been formulated into microspheres for sustained release. The present work aims towards studying the effect of combination of a natural chitosan from an oyster shell of Mystilis edulis and HPMC (hydroxy propyl methyl cellulose) (spectracel $15 \mathrm{E}$ ) as polymer and tripolyphosphate as cross linking agent using wet gelation technique. The various polymer combination ratios for different batches were compared with a low molecular weight standard chitosan. The extracted chitosan - HPMC polymer combination ratios were chosen at ten levels: as batches B1, B2, B3, B4, B5, B6, B7, B8, B9, B10 for 1:1, 1:2, 2:1, 1:0, 0:1, 3:1, 1:3, 5:1, and 1:5 and 1:1 having 450:450, 300:600, 600:300, 900:0, 0:900, 675:225, 225:675, 750:150, 150:750, 450:450 $\mathrm{mg}$ respectively, while the quantity of domperidone and tripolyphosphate remained constant. B11 and B12 were formulated with standard chitosan and HPMC. The percentage yield of the formulated microspheres was determined and then evaluated for flowability, drug entrapment efficiency, drug release and mechanism of drug release by Fickian diffusion. The best batches of the domperidone loaded microspheres produced from the combination polymer were compared with the standard chitosan. The highest yields of microspheres were given by batches B12, B11, B10, and B4 with values of $50.1 \pm 0.1 \%, 49.6 \pm 0.1 \%, 46.6 \pm 0.1 \%$, and $46.1 \pm 0.0 \%$ respectively while the lowest yield were $23.3 \pm 0.2 \%$ and $23.6 \pm 0.2 \%$. B5 and B6 and B9 did not yield any microsphere. The bulk density, tapped density, compressibility and Hausner's ratio of the microspheres showed good flowability and high percent compressibility. The drug entrapment efficiency showed that the entrapment ranged from 54.2 to 97.2 , where the least entrapment was B4 (54.2 \pm 0.1$)$ and the highest B12 (97.2 \pm 0.2$)$. The polymer surface of the microspheres as observed by SEM (scanning electron microscopy) was heterogeneous and porous which offers enhanced bioadhesivity. The dissolution study was used to determine the percentage drug release which ranged from $12.1 \%$ to $68.9 \%$ after 5 hours. Batches 1, 2, 3, 4, 7, and 11 follow zero order kinetics via Fickian diffusion. The results indicate that microspheres of domperidone could be successfully formulated with a natural chitosan either alone or in combination with HPMC for sustained delivery of domperidone. Furthermore, the concentration of the natural polymer and HPMC employed in the formulation need to be carefully selected to enable the production of microspheres with the desired sustained release properties.
\end{abstract}

Key words: Domperidone, HPMC, polymer combination, microspheres, wet gelation technique.

\section{Introduction}

Most crustaceans are delicacy consumed by many countries and races. They are available in the market in a wide variety of products. The sea food industries process and package the harvested products and the shells are generally left as waste. Even though the wastes are bio-degradable, the

Corresponding author: Stephen Olaribigbe Majekodunmi, $\mathrm{Ph} . \mathrm{D}$., research field: drug delivery in tropical diseases. dumping of large quantities makes degradation process low resulting in accumulation of waste over time which is a major environmental concern. Recycling of these shell waste and extraction of commercially viable substances like chitin and chitosan is an effective solution.

Chitin is the second most abundant polysaccharide on earth after cellulose [1]. It is a structural component of exo-skeleton of crustaceans, insects, mushrooms and cell wall of certain fungi and green algae. 
Chitosan, a biopolymer, is a linear polysaccharide composed of randomly distributed $\beta$-(1-4) length D-glucosamine and N-acetyl- D-glucosamine obtained by partial deacetylation of chitin and is structurally similar to cellulose which is composed of only monomer of glucose [2]. The main source of chitosan is from crustaceans waste, which is also the main cell wall material of most fungi [3, 4], cuticles of insects, yeast or green algae [5], crab and shrimp shells, oysters and lobsters [6].

Chitosan is considered one of the most valuable polymers for biomedical and pharmaceutical application due to its biodegradability, biocompatibility, antimicrobial, non-toxicity and antitumor properties. Nanoparticles, microspheres, hydrogel, films and fibers are typical chitosan based forms for biomedical and pharmaceutical application for several drug deliveries [7].

Kataria et al. [8] in their work defined microspheres as spherical micro particles in the diameter range of 1 and $1,000 \mu \mathrm{m}$. They are characteristically free flowing powders consisting of proteins or synthetic polymers. The range of techniques for the preparation of microspheres offers a variety of opportunities to control aspect of drug administration and enhance the therapeutic substance to the target site in a sustained control release fashion. It is a reliable means to deliver the drug to the target site with specificity.

DOM (domperidone) is a peripherally selective dopamine D2 receptor antagonist developed by Jansen pharmaceutica that is used as an antiemetic, gastroprokinetic agent and galactogue [9]. Domperidone does not readily cross the blood brain barrier and hence is less likely to cause central nervous system effect like sedation and dystonic reactions. It acts at the $\mathrm{CTZ}$ (chemo receptor trigger zone) and is unlikely to be effective in motion sickness and other vestibular disorders. It has a low ceiling anti-emetic and pro-kinetic action.

Domperidone is used for nausea and vomiting [10] associated with migraine [11], gastroparesis [12], in combination with levodopa in the management of Parkinson's disease [13], functional dyspepsia [14], in lactation acting as an antidopaminergic agent to promote lactation $[15,16]$.

Drug release studies of immediate release dosage forms in healthy subjects shows that domperidone tablets were not detectable in blood after few hours of oral administration and is eliminated in 5-7 hours from the body [17]. The dose of domperidone in adults greater than 16 is given as $10 \mathrm{mg} 3$ times daily.

Microspheres of domperidone will provide constant and prolonged therapeutic effect, reduce dosing frequency and thereby improve the patient compliance. Better drug utilization will improve the bioavailability and reduce the incidence or intensity of adverse effect and allow a controllable variability in degradation and drug release [18].

Thus, the aim of the study is to prepare sustained release of domperidone microspheres using a combination of locally extracted chitosan and HPMC (hydroxy propyl methyl cellulose) as polymers and tripolyphosphate as a cross linking agent to reduce the frequency of conventional dosage forms, side effects and improve adherence and health outcome.

\section{Materials and Methods}

\subsection{Materials}

Shells of Mystilis edulis were obtained from Long beach, California, USA, where they were seen scattered all over the beach. The extraction of chitosan from the Oyster shells of Mystilis edulis has done characterized elsewhere [19]. Domperidone is a gift from May and Baker PLC, Ikeja, Nigeria. HPMC (Spectracel 15E, low molecular weight) is a gift from Sensient, St. Louis, MO, USA. All other reagents and instruments are of analytical standard.

\subsection{Methods}

\subsubsection{Formation of Microspheres}

Microspheres were formed using the wet inversion technique in which $90 \mathrm{mg}$ of domperidone was 
Table 1 Formulation design.

\begin{tabular}{llllll}
\hline Batch no & Drug $(\mathrm{mg})$ & $\begin{array}{l}\text { Extracted chitosan } \\
(\mathrm{mg})\end{array}$ & $\begin{array}{l}\text { HPMC (spectracel } \\
15 \mathrm{E}(\mathrm{mg})\end{array}$ & $\begin{array}{l}\text { Extracted chitosan: } \\
\text { HPMC ratio }\end{array}$ & $\begin{array}{l}\text { Standard chitosan } \\
(\mathrm{mg})\end{array}$ \\
\hline B1 & 90 & 450 & 450 & $1: 1$ & - \\
B2 & 90 & 300 & 600 & $1: 2$ & - \\
B3 & 90 & 600 & 300 & $2: 1$ & - \\
B4 & 900 & 0 & $1: 0$ & - \\
B5 & 90 & 0 & 900 & $0: 1$ & - \\
B6 & 90 & 675 & 225 & $3: 1$ & - \\
B7 & 90 & 225 & 675 & $1: 3$ & - \\
B8 & 90 & 750 & 150 & $5: 1$ & \\
B9 & 90 & 150 & 750 & $1: 5$ & 750 \\
B10 & 90 & 450 & 450 & $1: 1$ & 150 \\
B11 & 90 & - & 150 & $5: 1$ & $1: 5$ \\
B12 & 90 & - & 750 & & \\
\hline
\end{tabular}

dissolved in $10 \mathrm{ml}$ of $2 \%$ acetic acid. The various polymer combination (chitosan and HPMC) at ratio $1: 1,1: 2,2: 1,1: 0,0: 1,3: 1,1: 3,5: 1$, and $1: 5$ and $1: 1$ having 450:450, 300:600, 600:300, 900:0, 0:900, 675: $225, \quad 225: 675, \quad 750: 150, \quad 150: 750, \quad 450: 450 \quad \mathrm{mg}$ respectively were added to the solution with gentle stirring. Only the best domperidone loaded microspheres produced from the various batches were compared with standard chitosan. The solution was left for 15 to $20 \mathrm{mins}$ to be bubble free. ТPP (Tripolyphosphate) $(3 \mathrm{~g})$ was dissolved in $50 \mathrm{~mL}$ of water. The combined polymer solution was slowly dropped into the TPP solution with the help of a 22-guage syringe. The microspheres formed were left in the TPP solution until they settled to the bottom of the container and additional 5 mins was allowed for sufficient crosslinking.

The TPP solution was decanted carefully and washed with acetone twice. The acetone was decanted and the microspheres were left for air drying. The dried microspheres were then collected. This process was repeated using different ratios of chitosan and HPMC.

\subsubsection{Percentage Yield}

The percentage yield of the prepared microspheres was determined after drying. The measured weight of prepared microspheres was divided by the total amount of all the non-volatile components used for the preparation of the microspheres.

$$
\text { yield }=\frac{\text { actual weight of product }}{\text { total weight of excipients }} \times 100
$$

\subsubsection{Drug Entrapment Efficiency}

Microspheres loaded with domperidone $(50 \mathrm{mg})$ were weighed accurately and crushed in a mortar. Powdered microspheres were suspended in $25 \mathrm{~mL}$ of $0.1 \mathrm{~N} \mathrm{HCl}$ for 10 mins. The solution was filtered and an aliquot was assayed spectrophotometrically at 278 $\mathrm{nm}$ to determine the drug content.

$$
\begin{gathered}
\text { Drug entrapment efficiency }= \\
\frac{\text { estimated drug content }}{\text { theoretical drug content }} \times 100
\end{gathered}
$$

2.2.4 Evaluation of Domperidone Loaded Microspheres

\subsubsection{Bulk Density}

Bulk density was determined using a graduated cylinder. The accurately weighed quantity of microspheres was added to cylinder and tapped three times. The volume was noted and the bulk density was calculated using the formula.

$$
\text { Bulk density }=\frac{M}{v}
$$

where $M=$ mass of sample, $V=$ volume of sample, $D \beta$ $=$ density

\subsubsection{Tapped Density}

Tapped density was determined using a graduated cylinder. The accurately weighed quantity of microspheres was added to the cylinder and tapped 
100 times. The volume noted, and tapped density was calculated using the formula.

$$
D o=\frac{M}{V p}
$$

where $M=$ weight of sample, $V_{P}=$ final tapped volume of microspheres, $D o=$ tapped density

2.2.4.3 Morphology of Domperidone Loaded Microspheres through SEM (scanning electron microscopy).

Shape and surface morphology of chitosan-HPMC domperidone microspheres were studied using Scanning Electron Microscope (SEM, JSM 6100, Jeol, Japan). Particle sizes of the microcapsules were evaluated using optical microscope were small amount of microspheres was placed on a slide and oil of immersion was dropped and then covered with a cover slide and then placed under the microscope and viewed.

2.2.4.4 In-vitro Drug Release Study of Microspheres

In-vitro drug release studies was carried out using USP 25 (type 11) apparatus in $400 \mathrm{~mL}$ of dissolution medium maintained at $37 \pm 1{ }^{\circ} \mathrm{C}$ at speed of $50 \mathrm{rpm}$. $\mathrm{HCl}(0.1 \mathrm{~N})$ was used as dissolution medium. Aliquots of $10 \mathrm{~mL}$ were withdrawn at predetermined time intervals using calibrated pipette during 3 hours period and filtered. An equivalent amount of a fresh dissolution medium maintained at $37 \pm 1{ }^{\circ} \mathrm{C}$ was added after withdrawing each sample to maintain the sink conditions. The drug concentration in the samples was analyzed spectrophotometrically at $278 \mathrm{~nm}$. The mean of four readings was used to determine concentration and percentage release of the drug.

\subsection{Calibration Curve of Domperidone}

As domperidone is poorly soluble in water, organic solvent is required to solubilize the drug. Therefore, stock solution of domperidone was prepared in methanol and working standards were prepared by suitably diluting with $0.1 \mathrm{M} \mathrm{HCl}$ and the absorbance were measured using UV-Vis.

Spectrophotometer (Labomedinc.) at $\lambda_{\max } 287 \mathrm{~nm}$ is against blank. Calibration curve was plotted on drug concentration $(20-120 \mu \mathrm{g} / \mathrm{mL})$.

2.2.4.4.2 Mathematical Model of Domperidone Release Profile

Domperidone release data were analyzed according to zero order kinetics, first order kinetics and Higuchi model to characterize the mechanism of drug release.

\subsubsection{Statistical Analysis}

Experimental data have been represented as the mean with SD (standard deviation) of different independent determinations. The significance of differences was evaluated by ANOVA (analysis of variance). Differences were considered statistically significant at $P<0.05$.

\section{Results and Discussion}

\subsection{Characterization of Microspheres}

\subsubsection{Percentage Yield}

The highest yield of microspheres were given by B12 (1:5), B11(5:1), B10(1:1), B4 (1:0) i.e. domperidone loaded microspheres formulated using $750 \mathrm{mg}$ HPMC and $150 \mathrm{mg}$ standard chitosan, $150 \mathrm{mg}$ HPMC and $750 \mathrm{mg}$ standard chitosan, $450 \mathrm{mg}$ extracted chitosan and $450 \mathrm{mg}$ HPMC and $(900 \mathrm{mg}$ of extracted chitosan alone) with values of $50.1 \pm 0.1 \%$, $49.6 \pm 0.1 \%, 46.6 \pm 0.1 \%$, and $46.1 \pm 0.0 \%$ respectively. The lowest yield were B6 and B9 i.e. domperidone loaded microspheres using $675 \mathrm{mg}$ extracted chitosan and 225 HPMC (ratio 3:1) and 150 mg extracted chitosan and $750 \mathrm{mg}$ HPMC with values $23.3 \pm 0.2 \%$ and $23.6 \pm 0.2 \%$ respectively while Batches B6 (3:1) i.e. $675 \mathrm{mg}$ extracted chitosan and $225 \mathrm{mg}$ of HPMC and B9 (1:5) i.e. $150 \mathrm{mg}$ extracted chitosan and $750 \mathrm{mg}$ HPMC and B5 (0:1) $(900 \mathrm{mg}$ HPMC only) did not yield any microsphere.

It was observed that the batches consisting of standard chitosan gave the highest yield. The batch consisting of higher amount of extracted chitosan as polymer and lesser amount of HPMC ratio gave higher yield than batches with higher amount of HPMC and less extracted chitosan. The low yield can 
Table 2 Percentage yield of domperidone microspheres.

\begin{tabular}{lllll}
\hline Batch no & $\begin{array}{l}\text { Chitosan } \\
(\mathrm{mg})\end{array}$ & $\begin{array}{l}\text { HPMC } \\
(\mathrm{mg})\end{array}$ & Polymer ratio & \%Yield \\
\hline B1 & 450 & 450 & $1: 1$ & $33.1 \pm 0.1$ \\
B2 & 300 & 600 & $1: 2$ & $32.3 \pm 0.1$ \\
B3 & 300 & $2: 1$ & $29.6 \pm 0.2$ \\
B4 & 900 & 0 & $1: 0$ & $46.1 \pm 0.0$ \\
B6 & 900 & 225 & $3: 1$ & $23.3 \pm 0.2$ \\
B7 & 675 & 675 & $1: 3$ & $32.6 \pm 0.2$ \\
B8 & 225 & 150 & $5: 1$ & $34.3 \pm 0.1$ \\
B9 & 750 & 750 & $1: 5$ & $23.6 \pm 0.2$ \\
B10 & 150 & 450 & $1: 1$ & $46.6 \pm 0.1$ \\
B11 & 450 & 150 & $5: 1$ & $49.6 \pm 0.1$ \\
B12 & 750 & 750 & $1: 5$ & $50.1 \pm 0.1$ \\
\hline
\end{tabular}

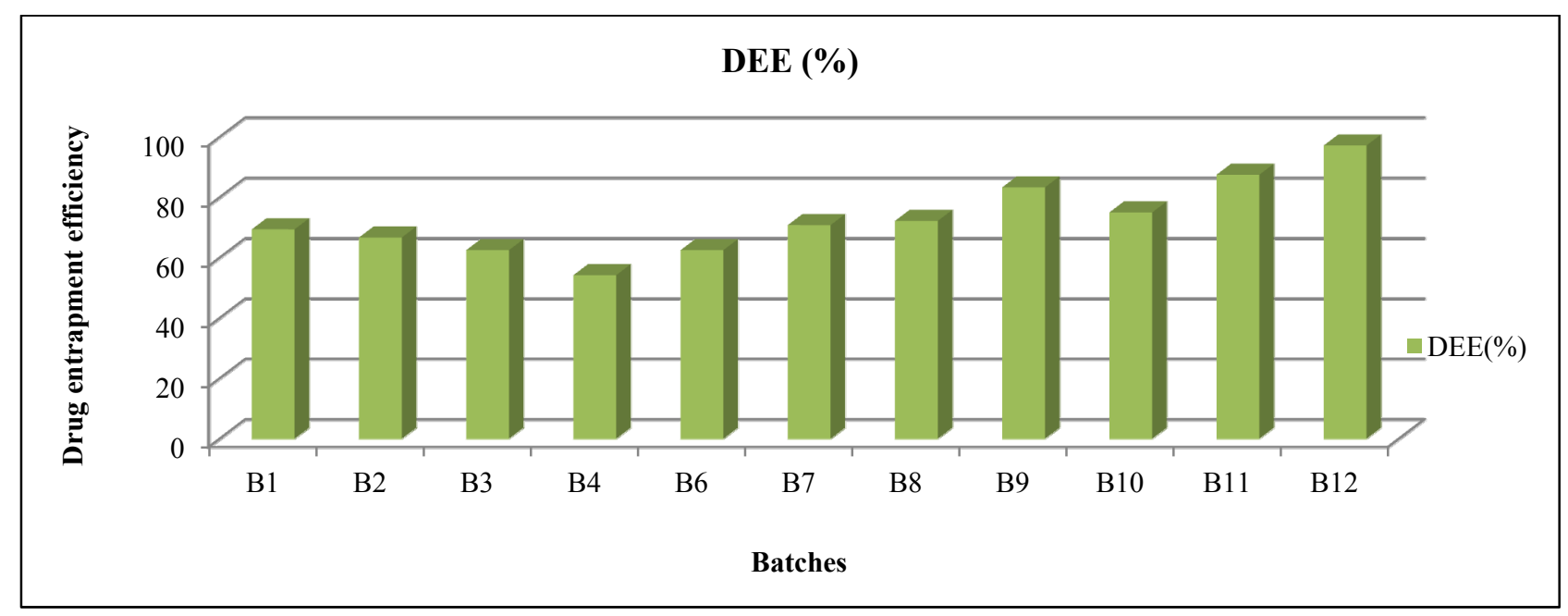

Fig. 1 Representation of the drug entrapment efficiency of all batches of domperidone microspheres.

be attributed to either the wastage of the formed microspheres during the washing and drying process, or the method used in the formation of microspheres, the cross linking time, concentration of the cross linking agent (tripolyphosphate) or amount of the incorporated drug. There was a significant difference $(p<0.05)$ among all the batches.

\subsubsection{Drug Entrapment Efficiency}

The drug entrapment efficiency showed that the entrapment efficiency ranged from $54.2 \%$ to $97.2 \%$. The least entrapment efficiency was B4 (1:0) (54.2 \pm $0.1)$ and the highest was B12 (1:5) $(97.2 \pm 0.2)$. The result showed that the batches consisting of the standard chitosan-HPMC polymer ratio gave a higher drug entrapment efficiency i.e. B10 (1:1), B11 (5:1) and B12 (1:5). B1 to B9 formulated using the extracted chitosan showed higher entrapment when the amount of the HPMC was increased compared to the corresponding batches with higher amount of chitosan. This shows a direct relationship between the amount of HPMC and the drug entrapment efficiency of the microspheres. As the concentration of HPMC increased the drug entrapment efficiency increased. Hemlata et al. [20] reported drug entrapment efficiency of domperidone microspheres to be between $46 \%$ and $74 \%$, which is in accordance to the result obtained in this study.

Other research works have reported higher drug entrapment efficiency. Xiao et al. [21] found the drug content in the microspheres to be between $98.3 \pm 2.1 \%$ to $99.3 \pm 2.8 \%$. Entrapment efficiency indicates the uniform distribution of drug in the microspheres. B3 
Table 3 Characterization of domperidone microspheres.

\begin{tabular}{llllll}
\hline Batch no & Bulk density $(\mathrm{g} / \mathrm{mL})$ & Tapped density $(\mathrm{g} / \mathrm{m})$ & Hausners ratio & Carr's index (\%) & $\begin{array}{l}\text { Drug entrapment } \\
\text { efficiency }(\%)\end{array}$ \\
\hline B1 & 0.44 & 0.55 & 1.25 & 20.0 & $69.4 \pm 0.1$ \\
B2 & 0.54 & 0.65 & 1.20 & 16.9 & $66.7 \pm 0.1$ \\
B3 & 0.84 & 0.98 & 1.16 & 14.3 & $62.5 \pm 0.1$ \\
B4 & 0.40 & 0.42 & 1.05 & 4.8 & $54.2 \pm 0.1$ \\
B6 & 0.78 & 0.93 & 1.19 & 16.1 & $62.5 \pm 0.1$ \\
B7 & 0.50 & 0.54 & 1.08 & 7.4 & $70.8 \pm 0.2$ \\
B8 & 0.36 & 0.38 & 1.06 & 5.3 & $72.2 \pm 1.3$ \\
B9 & 0.78 & 0.94 & 1.21 & 17.0 & $83.3 \pm 0.2$ \\
B10 & 0.39 & 0.41 & 1.05 & 4.9 & $75.0 \pm 1.0$ \\
B11 & 0.37 & 0.38 & 1.03 & 2.6 & $87.5 \pm 0.2$ \\
B12 & 036 & 0.37 & 1.03 & 2.7 & $97.2 \pm 0.2$ \\
\hline
\end{tabular}

(62.5 \pm 0.1$), \mathrm{B} 6(62.5 \pm 0.1)$ and $\mathrm{B} 7(70.8 \pm 0.2), \mathrm{B} 8$ $(71.53 \pm 1.33)$ showed no significant difference $(p=$ $0.05)$ among the batches. However there is significant difference $(p<0.05)$ among the other batches.

3.1.3 Results of Bulk Density, Tapped Density, Hausners Ratio and Compressibility Index

The results of the bulk density, tapped density, Hausners ratio and Carr's compressibility index and drug entrapment efficiency are shown in Table 3.

The bulk density, tapped density, Hausners ratio and compressibility index are used to determine the powder flow of powders and the results showed results ranging from excellent to fair flow property.

\subsubsection{In Vitro Release of Drug from Microspheres}

The dissolution study was used to determine the percentage drug release which ranged from $12.1 \%$ to $68.9 \%$ after 5 hours. Several techniques have been utilized to improve the dissolution of active pharmaceutical ingredient [22]. It has been reported by Nagarsenker et al., [17] that the dissolution profile of domperidone pure drug in an acidic medium of $\mathrm{pH}$ 1.2 buffer showed drug to dissolve to the extent of $91 \%$ in $2 \mathrm{~h}$. However, in an alkaline $\mathrm{pH}$ the dissolution of the drug was much slower, with as much as only $16 \%$ dissolved in $15 \mathrm{~min}$. in buffer of $\mathrm{pH}$ 6.8. As maintenance of sink conditions is an important requirement of dissolution media and drug release from the microspheres is the desirable rate limiting factor, dissolution studies were performed in
pH 1.2 buffers [23].

In-vitro release of domperidone from the chitosan-HPMC microspheres exhibited an initial burst between $7.2 \%$ and $35.6 \%$ of drug for all batches in $1 \mathrm{~h}$. The hydration rate of HPMC is as a result of its hydroxypropyl groups that produced strongly viscose gel that usually plays an important role in drug release especially at the onset of the release profile ) [24]. Hamlata et al. [20] reported the in-vitro release of domperidone from ethyl cellulose microspheres to have exhibited initial burst release with $23 \%$ of drug being released in $1 \mathrm{~h}$. They also added that this initial burst release is a common observation in the microspheres formulations due to the presence of drug particles on the surface of the microspheres. The burst effect seen during the in-vitro release from the microspheres was lower in case of microspheres prepared from higher concentration of the polymer [ 25 , 26].

After the burst release, all the domperidone microsphere formulations showed sustained release of the drug up to $5 \mathrm{~h}$. There was a retardation of release of the drug which will give a sustained release of about 12 hours and more as compared to the conventional domperidone which is effective only for 7-8 hours. The result also showed an indirect relationship between the amount of chitosan and the rate of release i.e. the batches with higher amount of chitosan showed lower percentage release and vice versa, except for B9 (polymer 
Extracted Chitosan and Hpmc as Polymers

combination ratio of 1:5 i.e. $150 \mathrm{mg}$ extracted chitosan and $750 \mathrm{mg}$ HPMC) which showed lower percentage release even with higher amount of HPMC.

Comparatively there is no significant difference $(\mathrm{p}$ $=0$ ) between the extracted chitosan and the low molecular weight standard chitosan as regards the release kinetics and sustained release of the drug from the microspheres.

3.1.5 Mechanism and Kinetics of Domperidone Release from Microspheres

Batches 1, 2, 3, 4, 7, and 11 follow zero order kinetics via Fickian diffusion. This implies that these batches are ideal for sustained release formulation. The release of drug (domperidone) from these formulations is independent on initial drug dose or concentration. B6 and B10 followed first order kinetics. First order kinetics is dependent on initial concentration and log of percentage (\%) drug remaining is directly proportional to time. The batches B8, B9 and B12 that have Higuchi model of release follow square root kinetics and therefore the release of the drug is dependent on the square root of time.

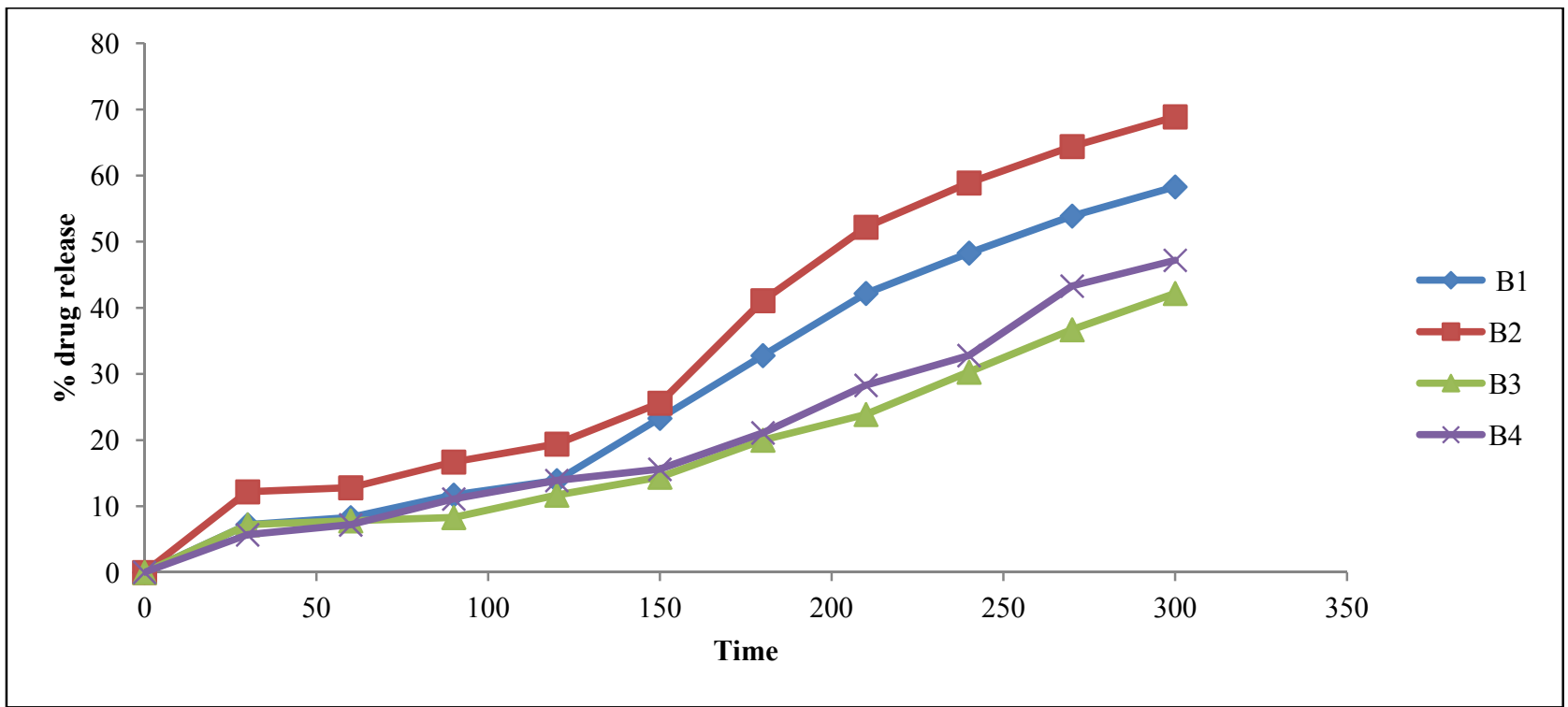

Fig. $2 \%$ drug release versus time of B1-B4.

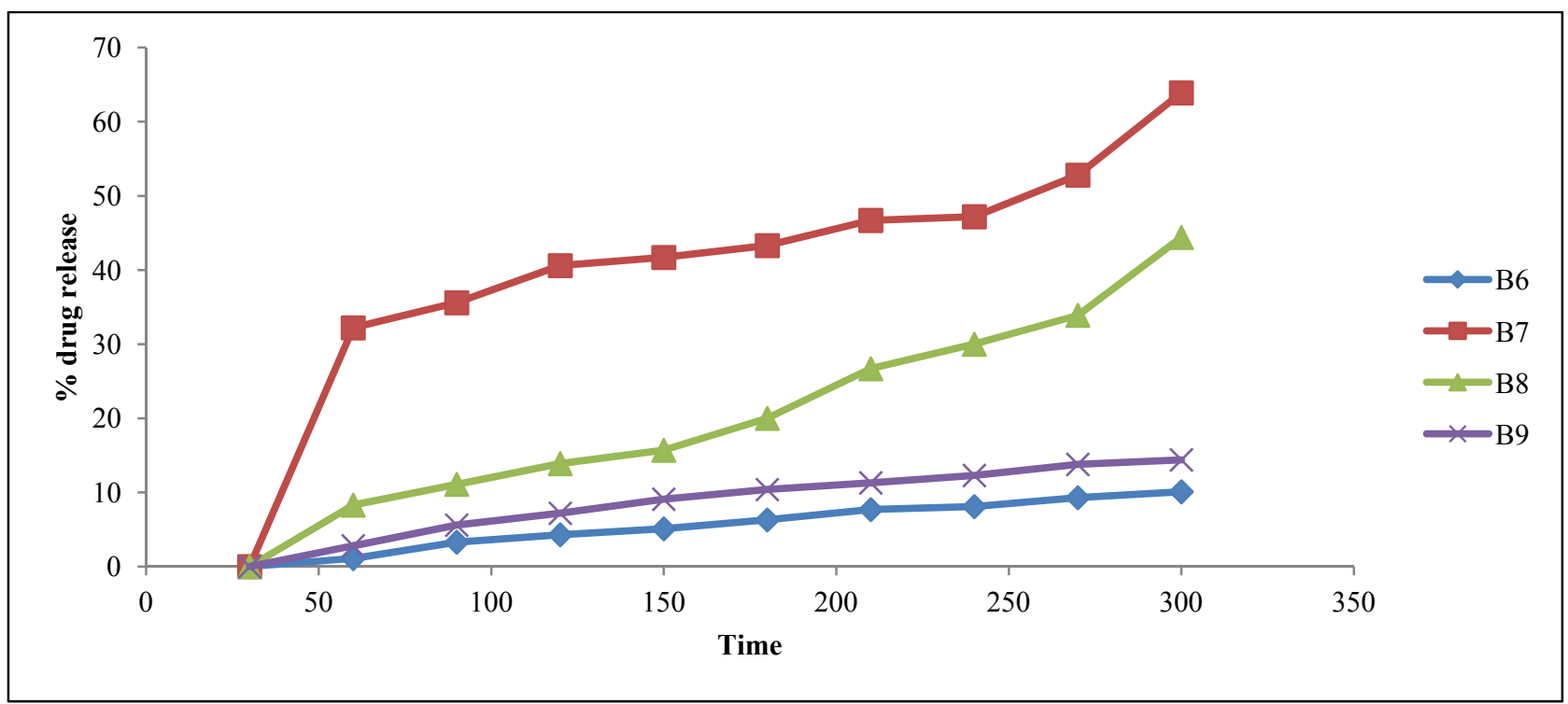

Fig. $3 \%$ drug release versus time of B6- B9. 


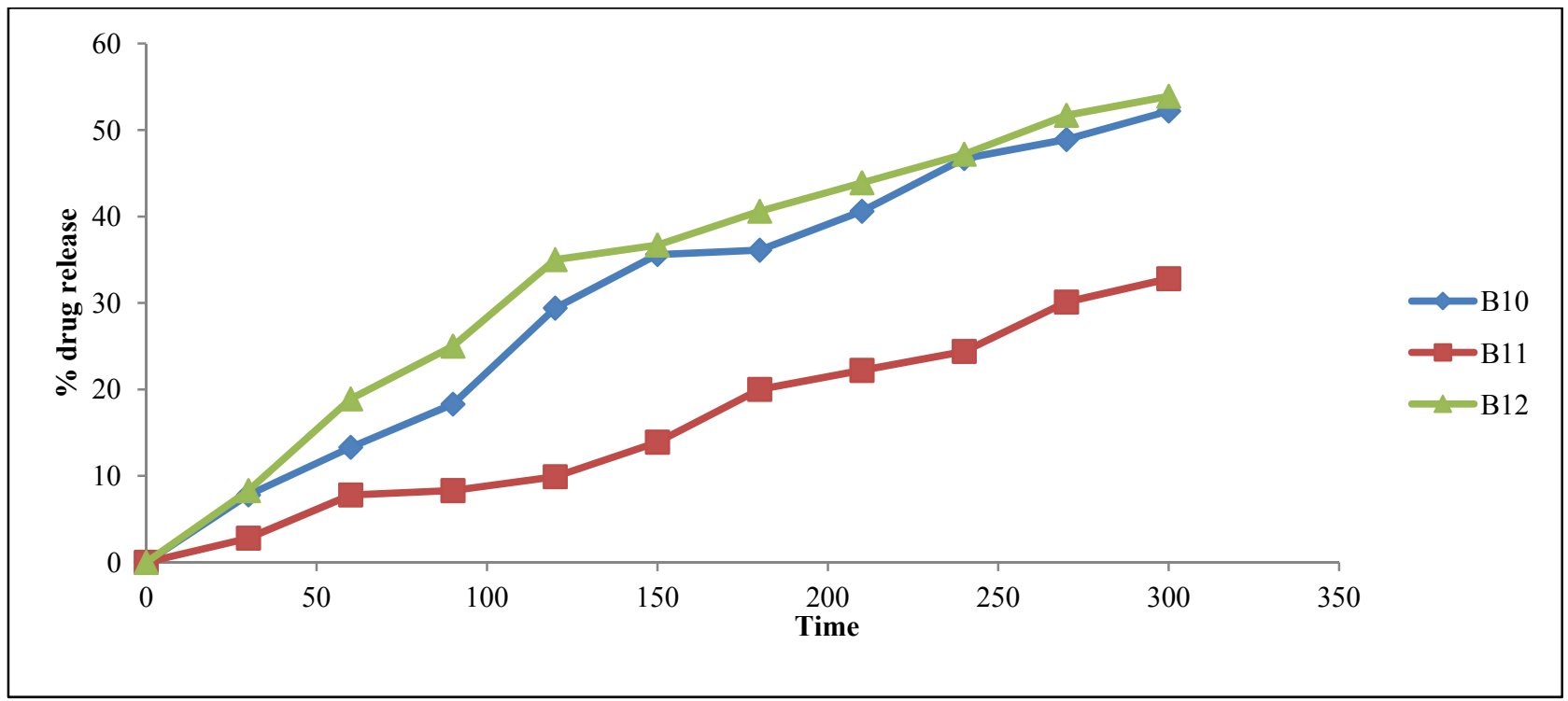

Fig. $4 \%$ drug release versus time of B10-B12.

However, the polymer concentration or method of incorporation of the polymer can be modified to alter the kinetics to zero order kinetics.

\subsubsection{Morphology}

The polymer surface of the microspheres as observed by SEM was heterogeneous and porous. These findings were similar with those of other researchers who reported rough surface morphologies for chitosan microspheres prepared by the orifice-ionic gelation technique [27, 28]. However, Kumbar et al. [29] reported smooth surface morphologies of chitosan microspheres when chitosan microspheres were produced in w/o emulsion and then cross-linked with glutaraldehyde. The surface morphology characteristics have an impact on bioadhesion. It has been found that the microspheres with a coarser and more porous surface may offer enhanced bioadhesivity as compared to those with a smoother texture [30]. Rough coarse surface observed in the present study might have led to bioadhesion. The surface morphology show the formation of cracks on the surface of the microspheres which may be due to the penetration of the dissolution medium into the microspheres and the subsequent dissolution of the drug and hence its diffusion through the polymer matrix (Figs. 5a and 6). SEM was also carried out for the domperidone powder as seen in Fig. 6.

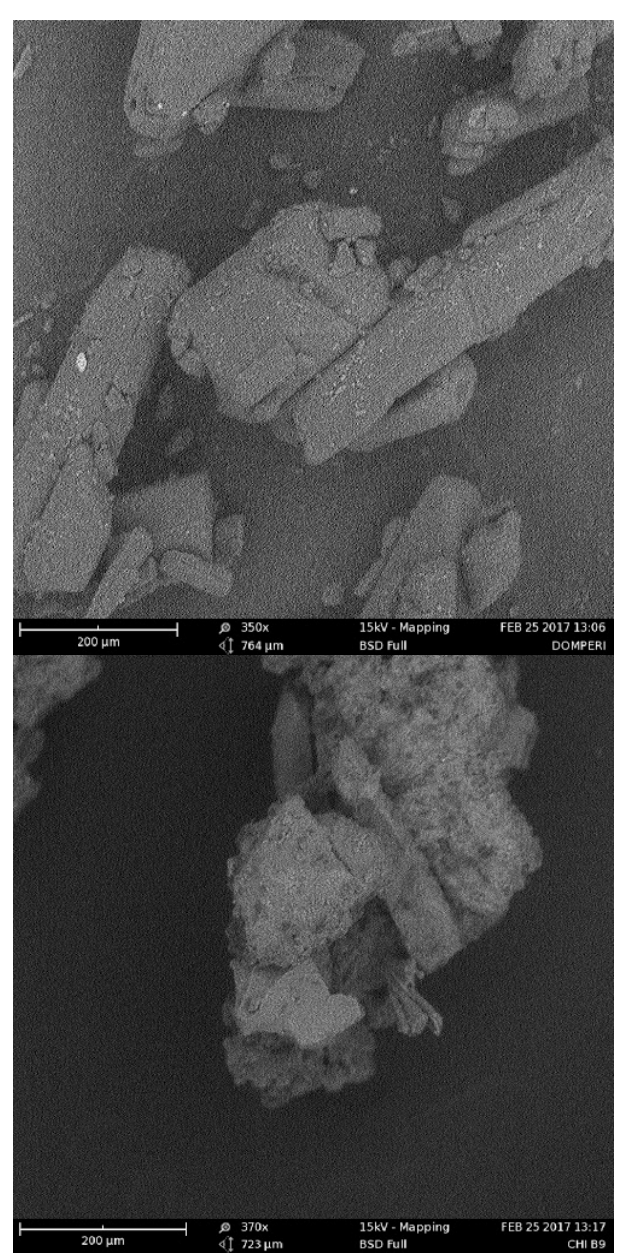

Fig. 5 SEM of a ( i.e. left) domperidone; b) domperidone loaded microspheres formulated with (B9) polymer combination ratio $1: 5$ i.e. $150 \mathrm{mg}$ chitosan from $M$. edulis and $750 \mathrm{mg}$ of HPMC). 


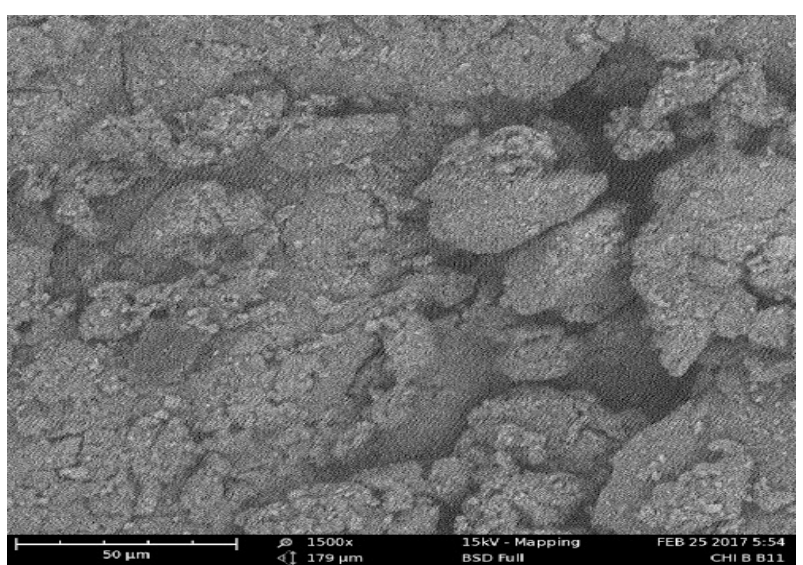

Fig. 6 SEM of domperidone loaded microspheres of B12 polymer combination ratio 1:5 i.e. $150 \mathrm{mg}$ standard chitosan and $750 \mathrm{mg}$ HPMC.

\section{Conclusion}

From the present study it is suggested that the extracted chitosan from Mystilis edulis and HPMC spectracel $15 \mathrm{E}$ are suitable candidates for sustaining the drug release from microspheres. The use of extracted chitosan from Mystilis edulis either alone or in low ratio with HPMC spectracel $15 \mathrm{E}$ as polymers for formulation of domperidone microspheres using wet gelation technique will be suitable for sustained release delivery of the drug. The developed technology is simple and can be easily scaled up and thus, holds enormous potential for commercial exploitation.

\section{References}

[1] Deshpande, S. S. 1995. "Structure-Digestibility Relationship of Legume 7s Proteins." J. Food Sci. 54: 108-13.

[2] Guibal, E., Touraud, E., and Roussy, T. 2004. "Chitosan Interactions with Metal Ions and Dyes: Dissolved-state. Solid-state Applications." World Journal of Microbioloical Biotechnology 21: 913.

[3] Williams, R., and Nicol, S. 1991. "Issues and Opportunities. Bureau of Rural Resources Proceedings 10. Bureau of Rural Resources and Australian Bureau of Agricultural." In Australian and New Zealand Southern Trawl Fisheries Conference: Southern Ocean Resources, 207-15.

[4] Tolaimate, A., Desbrieres, J., Rhazi, M., Alagui, M., Vincendon, M., and Vottero, P. 2000. "The Influence of Deacetylation Process on the Physicochemical
Characteristics of Chitosan from Squid Chitin.” Polymer 41: 2463-9.

[5] Kikkawa, T., Watanabe, Y., Katayama, Y., Kita, J., and Ishimatsu, A. 2008. "Acute $\mathrm{CO}_{2}$ Tolerance Limits of Juveniles of Three Marine Invertebrates, Sepia ly-cidas, Sepioteuthis Lessoniana, and Marsupenaeus Japonicus." Plankton Benthos Res. 3: 184-7.

[6] Wang, Q., Liu, Y., and Yan, H. 2007. "Mechanism of a Self-templating Synthesis of Monodispersed Hollow Silica Nanospheres with Tunable Size and Shell Thick-ness." Chem. Commun. 23: 2339-41.

[7] Kumar, M. N. V. R. 2000. "A Review of Chitin and Chitosan Applications." Reactive \& Functional Polymers (46): 1-27.

[8] Kataria, S., Middha, A., Sandhu, P., Ajay, B. and Bhawana, K. 2011. "Microsphere: A review." International Journal of Research in Pharmacy and Chemistry 1 (4): 1184-98.

[9] Barone, J. A. 1999. "Domperidone: A Peripherally Acting Dopamine2-receptor Antagonist." The Annals of Pharmacotherapy 33 (4): 429-40.

[10] Swann, I. L., Thompson, E. N., and Qureshi, K. 1979. "Domperidone or Metoclopramide in Preventing Chemotherapeutically Induced Nausea and Vomiting." British Medical Journal 2 (6199):1188.

[11] Worthington, I., Pringsheim, T., Gawel, M. J., Gladstone, J., Cooper, P., Dilli, E., Aube, M., Leroux, E., and Becker, W. J. 2013. "Canadian Headache Society Guideline: Acute Drug Therapy for Migraine Headache." The Canadian Journal of Neurological Sciences 40 (53): 356-67.

[12] Huang, W., Begum, R., Barber, T., Ibba, V., Tee, N., Hussain, M., Arastoo, M., Yang, Q., Robson, L., and Lesage, S. 2012. "Regenerative Potential of Silk Conduits in Repair of Peripheral Nerve Injury in Adult Rats." Biomaterials 33: 59-71.

[13] Nishikaw, N., Nagai, M., Tsujii, T., Iwaki, H., Yabe, H., and Nomoto, M. 2012. "Co-administration of Domperidone Increases Plasma Levodopa Concentration in Patients with Parkinson Disease." Clinical Neuropharmacology 35 (4): 182-4.

[14] Grzeskowiak, L. E., Lim, S. W., Thomas, A. E., Ritchie, U., and Gordon, A. L. 2013. "Audit of Domperidone Use as a Galactogogue at an Australian Tertiary Teaching Hospital." Journal of Human Lactation 29 (1): 32-7.

[15] Donovan, M. J., Kim, S. H., Raman, V., and Smyth, H. D. 2012. "Dry Powder Inhaler Device Influence on Carrier Particle Performance." J. of Pharm. Sci. 101 (3): 1097-107.

[16] Sakamoto, Y., Kato, S., Sekino, Y., Sakai, E, Uchiyama, T., Iida, H., Hosono, K., Endo, H., et al. 2011. "Effects of Domperidone on Gastric Emptying: A Crossover Study 
using a Continuous Real-time 13C Breath Test (Breath ID system)." Hepato-gastroenterology 58 (106): 637-41.

[17] Nagarsenker, M. S., Garad, S. D., and Ramprakash, G. 2000. "Design, Optimization and Evaluation of Domperidone Coevaporates." Journal of Control Release 63: 31-9.

[18] Fishers, TechNote. 2001. Working with Microspheres 9025 Technology, www.bangslabs.com IN 46038-2886 800.387.0672.

[19] Sakamoto, Y., Kato, S., Sekino, Y., Sakai, E., Uchiyama, T., Iida, H., Hosono, K., Endo, H., et al. 2011. "Effects of Domperidone on Gastric Emptying: A Crossover Study Using a Continuous Real-Time 13C Breath Test (Breath ID system).” Hepato-Gastroenterology 58 (106): 637-41.

[20] Hemlata, G., Patil, R. T., Michael, A. R., and Kamalinder, K. S. 2011. "Formulation and Development of Orodispersible Sustained Release Tablet of Domperidone." Journal of Pharmacy and Biomedical Sciences 34 (6): 246-56.

[21] Xiao, M., Qiu, X., Yue, D., Cai, Y., and Mo, Q. 2013. "Influence of Hippophaerhamnoides on Two Appetite Factors, Gastric Emptying and Metabolic Parameters, in Children with Functional Dyspepsia." Hellenic Journal of Nuclear Medicine 16 (1): 38-43.

[22] Tiong, N., and Elkordy, A. A. 2009. "Effects of Liquisolid Formulations on Dissolution of Naproxen." European Journal of Pharmacy and Biopharmacy 73: 373-84.

[23] Amidon, G. L., Lennernas, H., Shah, V. P., and Crison, J. R. 1995. "A Theoretical Basis for a Biopharmaceutic
Drug Classification: The Correlation of in-vitro Drug Product Dissolution and in-vivo Bioavailability." Journal of Pharmaceutical Resources 12 (3): 413-20.

[24] Arun, M., and Mahale, S. A. 2013. "Sreenivas N. Development and Characterization of Diltiazem Hydrochloride Microspheres." Journal of Pharmaceutical, Biological and Chemical Sciences 4 (2): 567-75.

[25] Dhankar, V. Garg, G, Dhamija, K., and Awasthi, R. 2014. "Preparation, Characterization and Evaluation of Ranitidine Hydrochloride-loaded Mucoadhesive Microspheres." Polim Med. Apr-Jun 44 (2): 75-81.

[26] Jameela, S. R., and Jayakrishnan, A. 1995. "Glutaraldehydecrosslinked-chitosan Microspheres as a Long Acting Biodegradable Drug Delivery Vehicle:studies on the in-vitro Release of Mitoxantrone and in Vivo Degradation of Microspheres in Rat Muscle." J. Control Rel. 16: 769-75.

[27] Shu, X. Z., and Zhu, K. J. 2000. "A Novel Approach to Prepare Tripolyphosphate/Chitosan Complex Beads for Controlled Drug Delivery." Int. J. Pharm. 201: 51-8.

[28] Govender, S., Pillay, V., Chetty, D. J., and Essack, S. Y. 2005. "Optimization and Characterization of Bioadhesive Controlled Release Tetracycline Microspheres." Int. J. Pharm. 306: 24-40.

[29] Kumbar, S. G., Kulkarni, A. R., and Aminabhavi, M. 2002. "Crosslinked Chitosan Microspheres for Encapsulation of Diclofenac Sodium: Effect of Crosslinking Agent." J. Microencapsul 19 (2): 173-80.

[30] Vasir, J. K., Tambwekar, K., and Garg, S. 2003. "Bioadhesive Microspheres as a Controlled Drug Delivery System.” Int. J. Pharm 255:13-32. 